\title{
Increasing relational memory in childhood with unitization strategies
}

\author{
Alison Robey ${ }^{1} \cdot$ Tracy Riggins $^{1}$
}

Published online: 28 August 2017

(C) Psychonomic Society, Inc. 2017

\begin{abstract}
Young children often experience relational memory failures, which are thought to result from immaturity of the recollection processes presumed to be required for these tasks. However, research in adults has suggested that relational memory tasks can be accomplished using familiarity, a process thought to be mature by the end of early childhood. The goal of the present study was to determine whether relational memory performance could be improved in childhood by teaching young children memory strategies that have been shown to increase the contribution of familiarity in adults (i.e., unitization). Groups of 6- and 8-year-old children were taught to use visualization strategies that either unitized or did not unitize pictures and colored borders. Estimates of familiarity and recollection were extracted by fitting receiver operator characteristic curves (Yonelinas, Journal of Experimental Psychology: Learning, Memory, and Cognition 20, 13411354, 1994, Yonelinas, Memory \& Cognition 25, 747-763, 1997) based on dual-process models of recognition. Bayesian analysis revealed that strategies involving unitization improved memory performance and increased the contribution of familiarity in both age groups.
\end{abstract}

Keywords Familiarity in recognition memory $\cdot$ Memory . Development $\cdot$ Memory strategies $\cdot$ Recollection

The ability to bind one piece of information to another and remember it across a delay (i.e., relational memory) is present in the first few years of life (e.g., Bemis \& Leichtman, 2013).

Alison Robey

arobey@umd.edu

1 University of Maryland, 1105G Biology-Psychology Building, College Park, MD 20740, USA
For example, young children are often tasked with remembering the route from their house to the bus stop or curriculum material such as learning relations between animals and their habitats. Although young children are expected to complete tasks such as these successfully, they often fail. One possible explanation for these failures is they occur due to the immaturity of basic relational memory abilities (Sluzenski, Newcombe, \& Kovacs, 2006).

Historically, relational memory abilities have been thought to rely on recollection, one of the two cognitive processes argued to underlie recognition memory (Yonelinas, 2002). During childhood, recollection has been shown to follow a protracted developmental trajectory into the adolescent years (Ghetti \& Bauer, 2012). However, it has recently been suggested in the adult literature that if two pieces of to-beremembered information are bound in a unitized fashion (i.e., "fused" into a single item), the contribution of another cognitive process, familiarity, is significantly increased (Yonelinas, Kroll, Dobbins, \& Soltani, 1999). This is a particularly exciting possibility from a developmental perspective, because familiarity is thought to reach maturity before recollection. Specifically, if children unitize to-be-remembered pieces of information, they may be less prone to errors and forgetting as a result of increased reliance on relatively "mature" familiarity processes.

The goal of the present study was to take what is known about manipulations that increase the contribution of familiarity to relational memory processes in adults and determine whether the same memory strategies would improve children's performance on a relational memory task. Because this study relies heavily on a dual-process theory of memory, we will first review this approach. Then the concept of unitization will be discussed followed by a review of stimuli manipulations that make use of unitization phenomena and how unitization may be used as a strategy through visual imagery. 
Finally, a brief review of memory development will be used to shed light on why a visual unitization strategy might improve relational memory performance during early childhood.

\section{A dual-process theory of memory}

Yonelinas (2002) proposed that recognition memory is a dualprocess system, composed of two independent cognitive processes: familiarity and recollection. Familiarity is reflected as a global feeling of knowing, whereas recollection requires the remembering of specific contextual details surrounding an event. Many differences are known to exist between these processes (see Yonelinas, 2002, for a review), with one of the most apparent being their ability to support the learning of novel relations, as in relational memory tasks (Diana, Reder, Arndt, \& Park, 2006).

It was originally thought that familiarity could support performance on tasks requiring the recognition of a single item (i.e., item memory tasks), but that recollection was required when memory for two items was required (i.e., relational memory tasks). However, recent work has shown that familiarity is able to support relational memory if the to-beremembered information is encoded in a coherent gestalt fashion (i.e., if it is unitized; Yonelinas et al., 1999). For example, Yonelinas et al. (1999) presented participants with faces either right-side up or upside down and found that upright faces, which are processed holistically, could be recognized through the use of familiarity, whereas upside-down faces, which are processed as separate, individual features and therefore require binding, could not be retrieved with familiarity alone. Thus, whether or not familiarity can support memory for relations between items is dependent on the way the items are initially processed (Diana, Yonelinas, \& Ranganath, 2008).

The process of encoding separate items together as a single unit is referred to as unitization. Recent theories consider unitization to be a continuum, with differing levels of unitization ranging from high (i.e., multiple pieces of information completely combined) to low (i.e., multiple pieces of information completely separated; Parks \& Yonelinas, 2015). Unitization allows for better recognition because it increases the contribution of familiarity, however evidence is mixed regarding whether unitization has any impact on recollection (Diana, Ven den Boom, Yonelinas, \& Ranganath, 2011; Parks \& Yonelinas, 2015).

\section{Unitization increases the contribution of familiarity}

Many methods have been used to show that the contribution of familiarity is increased when items are unitized relative to when items are not (Ecker, Maybery, \& Zimmer, 2013; Giovanello, Keane, \& Verfaellie, 2006; Kan et al., 2011;
Kuo \& Van Petten, 2008; Mitchell, Johnson, Raye, \& Green, 2004; Quamme, Yonelinas, \& Norman, 2007; Rhodes \& Donaldson, 2007, 2008; Wilton, 1989). Improvements in memory due to unitization can occur through both stimulus manipulations (Ecker et al., 2013; Kan et al., 2011; Kuo \& Van Petten, 2008; Mitchell et al., 2004; Wilton, 1989) and manipulations of the task encoding instructions (Giovanello et al., 2006; Quamme et al., 2007; Rhodes \& Donaldson, 2007; Tibon, Gronau, Scheuplein, Mecklinger, \& Levy, 2014). Since manipulations involving task instructions are most relevant to the present study, they are elaborated upon below.

Semantic binding with unitization Most relevant to the present study, the holistic processing of to-be-remembered information can occur with semantic binding, such as manipulations to the processing of word pairs (Giovanello et al., 2006; Quamme et al., 2007; Rhodes \& Donaldson, 2007). Giovanello et al. found that when word pairs were presented as either compound (e.g., RAIN-BOW) or unrelated (e.g., RAIN-FORK) word pairs, participants were better able to remember the compound word pairs. Holistic processing of novel words pairs can also be induced under certain manipulations (Quamme et al., 2007). When presented with novel word pairs such as CLOUD-LAWN, participants were given sentences that either promoted holistic processing (e.g., a cloud lawn is a grassy area used for sky gazing) or kept the words separate (e.g., while the boy lay on the lawn, he looked up at the clouds). When holistic encoding of the word pairs was encouraged, the contribution of familiarity increased during recognition; however, memory performance was equal across conditions.

Unitization as a strategy Familiarity can also be heightened through unitization in the form of participant-initiated strategies (Bastin et al., 2013; Diana et al., 2008; Rhodes \& Donaldson, 2008). It has been shown, in adults, that visual imagery is a useful memory strategy that improves memory performance on relational memory tasks more than overt strategies such as rehearsal (Bower, 1970; McGee, 1980). Visual imagery has been used to promote unitization and in doing so, increase familiarity in nonunitized stimuli. It should be noted that visualization strategies that promote unitization makes use of levels of unitization (LOU) and specifically target familiarity and associative memory, as opposed to elaborative encoding strategies, which are known to improve item memory (see levels of processing; Craik, 2002; Parks \& Yonelinas, 2015). Two primary visualization strategies have been used to show an increase in the contribution of familiarity through strategies that promote high unitization: (1) imagining a stimulus as a certain color and (2) imaging two stimuli interacting with each other. First, Diana et al. (2008) tasked participants with remembering a word and the background color on which 
it was presented. Participants in the unitized condition were instructed to visualize a situation in which the item would be the color of the background. In contrast, participants in the nonunitized condition were instructed to visualize the item associated with another item the color of the background (e.g., a stop sign if the background was red and a dollar bill if the background was green). The results showed that although no statistical difference was observed in the ability to discriminate old from new items or the background color of the items between conditions, participants in the unitized condition showed a significant increase in the contribution of familiarity to recognition, as shown by receiver operating characteristic (ROC) curves (see also Bastin et al., 2013). Similarly, Rhodes and Donaldson (2008) tasked participants with remembering both associated and unassociated word pairs. Within their unitization condition, participants were told to use an interactive imagery strategy, whereas in their nonunitized condition the participants were told to visualize both words separately. In contrast to Diana et al.'s (2008) results, Rhodes and Donaldson (2008) found that not only was familiarity increased for participants in the unitized relative to the nonunitized condition, participants also performed better on the recognition memory task.

\section{Memory development}

Previous research has shown that performance on relational memory tasks improves throughout childhood (Bemis \& Leichtman, 2013; Drummey \& Newcombe, 2002; Fandakova, Shing, \& Lindenberger, 2013; Lloyd, Doydum, \& Newcombe, 2009; Lorsbach \& Reimer, 2005; Riggins, 2014; Scarf, Gross, Colombo, \& Hayne, 2013; Yim, Dennis, \& Sloutsky, 2013). For example, when tasked with remembering items, backgrounds, and item + background combinations, 4-, 6-, and 8-year-old children showed no differences in their abilities to remember items or backgrounds. However, the ability to remember item + background pairs, improved with age (Sluzenski et al., 2006). Likewise, when similar aged children were tested on a novel fact paradigm that required remembering new facts along with who taught the facts, no age-related differences were observed in memory for the facts, but age-related improvements were observed in memory for who taught the facts (Drummey \& Newcombe, 2002). In fact, a subsequent longitudinal study examining change between 4 and 10 years pinpointed the period between 5 and 7 years of age as showing the most dramatic improvements in relational memory (Riggins, 2014). Gradual improvements continue into adolescence, particularly on difficult relational memory problems such as binding multiple items to locations on a grid (Lorsbach \& Reimer, 2005).

The results of the studies above exploring the development of relational memory are consistent with those from studies exploring the development of familiarity and recollection, which suggest earlier maturation of the former than of the latter. Billingsley, Smith, and McAndrews (2002), used a remember/know paradigm and observed changes in recollection from childhood (8-10 years of age) to adulthood, but no changes in familiarity. Likewise, in two conjoint-recognition studies exploring the development of familiarity and recollection, it was found that from early to late childhood (5 to 11 years of age) recollection improved, but familiarity did not change (Brainerd, Holliday, \& Reyna, 2004). When testing 6-, 8-, 10-, 12-, and 14-year-old children using ROCs, Ghetti and Angelini (2008) observed that recollection improved across all age groups, whereas familiarity did not (when sufficient processing time was given; see Ghetti \& Angelini, 2008 , for details). This supports the notion that familiarity is relatively mature by early childhood (i.e., 6 years of age), whereas recollection continues to develop into adolescence (Brainerd et al., 2004; Ghetti \& Angelini, 2008).

Together, the studies above suggest a protracted development of recollection between middle childhood and adolescence, but relative maturity of familiarity during this time. It is possible that if a mechanism was used to increase reliance on familiarity during this period, it might improve children's relational memory by increasing the contribution of this relatively more mature process. One proposed mechanism is unitization through visual imagery. Below we will discuss how unitization has been used to improve relational memory performance in populations similar to children.

\section{Increasing memory performance in special populations}

It is reasonable to suggest that unitization may boost relational memory performance (by increasing the contribution of familiarity) in young children, who do not have fully developed recollection, because these strategies have been shown to improve performance in populations in which recollection has been compromised. First, multiple studies have shown that unitization can increase relational memory performance in patients with brain damage due to various causes (Quamme et al., 2007; J. D. Ryan, Moses, Barense, \& Rosenbaum, 2013); however, it should be noted that these manipulations only worked if the brain areas responsible for familiarity remained intact (Quamme et al., 2007). Additionally, it is well documented that elderly adults experience declines in episodic memory especially in tasks requiring binding (for a review, see Naveh-Benjamin, 2000), and elderly adults have also been shown to benefit from unitization strategies (Bastin et al., 2013; Zheng, Li, Xiao, Broster, \& Jiang, 2015; Zheng, Li, Xiao, Ren, \& He, 2016). 


\section{Unitization in childhood}

On the basis of research in older adults showing improvements in relational memory performance, younger children might also benefit from a unitization strategy on relational memory tasks. Specifically, the hypothesis is that because familiarity has been shown to reach maturity earlier in development, unitization strategies might improve children's relational memory due to increased reliance on familiarity processes. The direct question has not been empirically tested; however, some evidence provides indirect support for this hypothesis. For example, bottom-up perceptual unitization has been observed in children as young as 5 years of age (Hale \& Piper, 1973; Spiker \& Cantor, 1980). Although memory strategies show significant development from the preschool to elementary school years, there is also support for the notion that children as young as 4 years of age can utilize memory strategies after training (for a review, see Schneider \& Sodian, 1997). For visual imagery strategies specifically, children as young as 5 years of age have been shown to be capable of using visual imagery strategies after training, improving their memory performance (Ryan, Ledger, \& Weed, 1987).

\section{Present study}

The goal of the present study was to determine whether using a visual unitization strategy would improve performance on an associative memory task in children. Although unitization strategies have been used to boost relational memory performance in adult populations, this effect has not yet been tested in children. Two groups of children, 6-year-olds and 8-yearolds, were brought to the lab. These age groups were chosen because their familiarity processes are thought to be mature; however, the age groups differ in that recollection is thought to be more mature in 8- than in 6-year-olds (Ghetti \& Angelini, 2008). The children were presented with pictures of common animals and items printed in black and white surrounded by either a red or a yellow border. The encoding strategy was manipulated between groups to limit carryover effects from one strategy to the other. Two groups were trained in visual unitization strategies (the unitized group and the interactive group, following Diana et al., 2011; Diana et al., 2011; and Rhodes \& Donaldson, 2008), and a third group was trained on a separate visualization strategy (non-unitization group). The non-unitization condition promoted elaborative encoding of the stimulus but did not promote integration of the stimulus and color, so it was expected that this condition would rely heavily on recollection. During retrieval, the children were shown only the black-and-white images; they were asked to remember whether the image's border was red or yellow and to make a confidence judgment about that decision. The confidence judgments were used to construct ROC curves and to determine the relative contributions of familiarity and recollection for each group. Children have been shown to make accurate self-memory judgments by 5 years of age, and ROC curves have been constructed to observe the relative contributions of familiarity and recollection for children as young as 6 years of age (Ghetti \& Angelini, 2008; Roebers, Gelhaar, \& Schneider, 2004). We predicted that relational memory would be improved by the visual unitization strategies (unitized and interactive conditions), due to the increased contribution of familiarity, which was thought to be mature in both groups.

\section{Method}

\section{Participants}

A total of 127 six- and eight-year-old children were recruited from the University Infant and Child Studies Database. The children were assigned to one of the three experimental conditions (see Table 1). This sample size was determined on the basis of a power analysis using parameter estimates based on the results of Diana et al. (2008). The data from eight children were excluded due to computer errors $(n=3)$, noncompliance $(n=2)$, failing the practice $(n=2)$, and not using the confidence scale $(n=1)$. It should be noted that collection for the non-unitization condition began after collection to the unitized and interactive conditions.

To ensure that all children were capable of understanding the task instructions and completing the task, participants with known developmental disorders, who were colorblind, or who heard English less than 50\% of the time were excluded from participation. Parents provided informed consent for all participants, and the 8-year-old children also provided written assent. All children received a small gift for participating.

\section{Materials}

Training stimuli Four black-and-white images from the Snodgrass and Vanderwart (1980) line drawings were used for the training portion of the study. These images are standardized, contain common objects and animals, and have frequently been used with children similar in age to those in the present study (e.g., Cycowicz, Friedman, \& Duff, 2003; Lloyd et al., 2009). The images were printed on standard-stock paper. Two of the images were surrounded by a red border, and two were surrounded by a yellow border. Colored images of a red apple and a yellow school bus were laid on the desk within all children's view. Red and yellow crayons were provided to ensure that the children understood the task.

Encoding stimuli An additional 120 black-and-white images from the Snodgrass and Vanderwart (1980) line drawings 
Table 1 Participant demographics

\begin{tabular}{lll}
\hline & Mean Age in Years $(S D)$ & $\begin{array}{l}\text { Total } N \\
(\% \text { Male })\end{array}$ \\
\hline $\begin{array}{lll}\text { 6-year-olds } \\
\text { Unitized }\end{array}$ & $6.59(0.37)$ & $19(57.9 \%)$ \\
Interactive & $6.41(0.28)$ & $21(42.9 \%)$ \\
Non-unitization & $6.29(0.24)$ & $19(36.8 \%)$ \\
8-year-olds & & $21(61.9 \%)$ \\
Unitized & $8.28(0.20)$ & $19(36.8 \%)$ \\
Interactive & $8.38(0.33)$ & $20(35.0 \%)$ \\
Non-unitization & $8.36(0.29)$ & \\
\hline
\end{tabular}

were used for the encoding portion of the task. All images were presented on a computer monitor to keep exposure as consistent as possible between subjects. Half of the images were surrounded by a red border, and half by a yellow border. All images were adjusted to be similar in size and as centered as possible within the border. The image-color pairings were counterbalanced between subjects and presented in a random order. Stimulus presentation and randomization was programmed with E-Prime 2.0 (Psychology Software Tools).

Retrieval During the retrieval portion, the same Snodgrass and Vanderwart (1980) images displayed during encoding were shown again, except without the colored borders. A 3point smiley face confidence scale was presented at the bottom of the screen to aid children in their confidence decisions. Similar scales have been used in previous research with children of this age and have been shown to aid in helping children make accurate memory judgments (Roebers et al., 2004). To ensure consistency across participants, all verbal responses were recorded by the experimenter with a standard keyboard.

\section{Procedure}

This study took place in one session that lasted approximately an hour and a half. The session consists of three portions: (1) training to ensure that the participants understood the task, (2) encoding, and (3) retrieval. The procedure was modified from the methods of Diana et al. (2008) to be appropriate for use with children. Between the encoding and retrieval portions, participants received a 10-min snack break. The study was explained to the participants as a storytelling exercise or a fact-telling exercise. The University Institutional Review Board approved all of the following methods.

Training To ensure that participants fully understood the directions of the task, they participated in a brief training session. First, participants were trained how to visualize. They were instructed to close their eyes and picture in their mind a red apple. They were told basic features of an apple to aid the visualization process, and then opened their eyes to find a printed image of an apple. Participants were asked to confirm that what they had pictured in their minds looked similar to the image. This process was repeated with a yellow school bus.

Once successful visualization of the red apple and the yellow school bus was established, participants were trained on the specific visualization instructions for their randomly assigned condition. All participants were presented with four training stimuli: an elephant with a red border, a shirt with a yellow border, a yoyo with a red border, and a butterfly with a yellow border. The participants in the unitized condition were instructed to "Come up with a story for why the picture would be the color of the border (i.e., red/yellow)." They were informed that their stories did not need to be realistic and that they could be as creative as they wanted. Children who struggled during the practice were provided with example stories to use as a guide. After the children had provided a story, they were told to visualize the story in the same manner in which they had visualized the apple and school bus. Participants were asked what color the item they were picturing was. Then they were given the red and yellow crayons and asked to color the printed stimulus the way they pictured it. Participants passed the practice trial if they completed coloring in the image with the correct color. Participants were given feedback if they did not fully color in the image. This process was repeated for all four training items. If by the end of the four trials participants had not correctly colored any of the stimuli, they were excluded from the analysis.

The participants in the interactive group completed the same training as those in the unitized group, with the exception that they were instructed to "come up with a story for why the pictured item would be interacting with another item the color of the border." The specific items for the red and yellow border were always a red apple and a yellow school bus. After the child had told their story, they were told to visualize their story like they had with the apple and school bus during training. Participants were then given red and yellow crayons to color in the printed stimulus the way they pictured it (i.e., they were expect to draw either the apple or school bus next to the stimulus). Participants were corrected if they did not draw the correct interacting item near the stimulus. As in the unitized condition, this process was repeated for all four training items, and if by the end of the four trials participants had not drawn the correct item near the stimuli, they were excluded from the analysis.

The participants in the non-unitization group had similar training to those in the unitization groups, with the exception that they did not complete a storytelling task. Instead, participants in the non-unitization group were instructed to generate facts about the picture on the screen. All participants told either one or two facts. For example, for the target image of an elephant, children produced facts such as "Elephants are big and have big ears" or "They can squirt water at you." After the children told their facts, they were told to close their eyes and to 
visualize first the picture and then either an apple or a school bus, depending on the color of the border. This condition was designed to keep the encoding time, verbal generation, and visualization of both the picture and color as similar as possible to the other conditions, with the exception that the picture and color were not integrated. During the coloring phase the children in this condition were instructed to draw what they visualized after the picture. Children were corrected if they did not draw either the apple or the school bus. These tasks kept the non-unitization condition as similar as possible in verbal generation, focus on the pictured item, and visualization, they just did not complete any tasks that would cause unitization between the picture and the color. Again, this process was repeated for all 4 training items and no children failed the training task in the non-unitization condition.

Encoding After the training portion, participants began the encoding portion of the experiment. Participants were presented with stimuli from the same image set as those viewed during the training portion. The images remained on the screen for the length of time it took the participants to come up with their story or give their facts, in order to reduce the cognitive load during encoding (see Ghetti \& Angelini, 2008, for the rationale). Similar to the training, participants were instructed to come up with either a story or facts, based on their condition, and then complete their specific visualization task. Once the story and visualization were complete, the experiment moved on to the next stimulus. If children provided stories that were not appropriate for their given condition, they were corrected by the experimenter and asked to try again. ${ }^{1}$ This process continued for 120 stimuli.

Retrieval The retrieval portion began approximately $10 \mathrm{~min}$ after the encoding portion had ended. Participants again viewed the images they saw during encoding, but during retrieval all images were presented with no border. Participants were first instructed to respond whether the image was originally presented with a red or yellow border. Once the color judgment had been made, participants rated their confidence of that judgment on a 3-point scale. A smiley face scale was be provided to aid the children in their judgments. A happy face represented a 3 , very confident, a neutral face represented a 2 , a little confident, and a sad face represented a 1 , not confident or guessing. Participants were encouraged to use the entire scale in their judgments.

\footnotetext{
${ }^{1}$ Children rarely needed to be corrected. Fewer than ten children were ever corrected, and the two children who consistently gave incorrect stories were removed from analyses and labeled as noncompliant.
}

\section{Data analytic approach and results}

All analyses consisted of a model comparison approach utilizing Bayes factors within the analysis of variance (ANOVA) framework (Rouder, Morey, Speckman, \& Province, 2012). This approach provides benefits beyond null hypothesis significance testing (NHST) approaches, because opposed to a dichotomous decision, Bayes factors provide an index of how well the data support one of two competing hypotheses, allowing for claims to be made regarding both effects and lack of effects, with only the former claim able to be made using NHST. Bayes factors fall along a continuum with values greater than 1 represent greater support for the more complex (i.e., alternative) hypothesis, whereas Bayes factors less than 1 represent greater support for the more simple (i.e., null) hypothesis. The approach does not included thresholds for decision making and instead values further away from 1 are thought to represent greater support for that particular hypothesis. However, it is generally accepted that Bayes factors (BFs) between 0.33 and 3 (representing ratios of 3 to 1 ) do not provide enough evidence for either hypothesis to make a strong claim (Jeffreys, 1961). For our NHST results, go to https://osf.io/fb7vk/.

All analyses were run using the BayesFactor package in $\mathrm{R}$ and used the default prior, which this package defines as a distribution of prior probabilities of effect sizes using the Cauchy distribution centered at 0 , originally suggested by Jeffreys (1961). This prior represents the belief that potential observed effect sizes are more likely to be small than large. For comparison utilizing a null effect, it was defined as a point null $=0.0$. Additional sensitivity analyses were run to determine the impact of widening the priors on outcomes; however, no outcomes changed, and therefore only the results with the default priors are presented.

\section{Differences in memory performance}

A measure of children's ability to discriminate the correct from the incorrect color $\left(d^{\prime}\right)$, regardless of confidence rating, served as the dependent variable for the comparison between groups. For all analyses, $d^{\prime}$ was calculated with red as the target color. Main effects of age and condition were compared against a null model, and their interaction was compared against a model with both main effects.

We found decisive support for a main effect of condition $\left(\mathrm{BF}_{10}=8.41 \times 10^{18}\right)$. Follow-up analyses revealed support for memory performance being greater for both the unitized and interactive conditions than for the non-unitization condition $\left(\mathrm{BFs}_{10}=7.65 \times 10^{15}, 2.30 \times 10^{15}\right)$. The results, however, were inconclusive regarding differences between the unitized and interactive conditions $\left(\mathrm{BF}_{10}=1.72\right)$. Data were also inconclusive regarding differences in age $\left(\mathrm{BF}_{10}=0.36\right)$ and Age $\times$ Condition interaction $\left(\mathrm{BF}_{10}=0.61\right)$. 
Table 2 Proportions of color correct and $d$ 's for both age groups, $M$ $(S D)$

\begin{tabular}{lll}
\hline & Color Correct & $d^{\prime}$ \\
\hline 6-year-olds & & \\
Unitized & $.75(.10)$ & $1.44(0.65)$ \\
Interactive & $.78(.11)$ & $1.60(0.72)$ \\
Non-unitization & $.52(.06)$ & $0.10(0.29)$ \\
8-year-olds & & \\
Unitized & $.77(.10)$ & $1.57(0.65)$ \\
Interactive & $.83(.13)$ & $2.19(0.97)$ \\
Non-unitization & $.52(.08)$ & $0.13(0.42)$ \\
\hline
\end{tabular}

In light of suggestions from past developmental work (Lloyd et al., 2009), the proportions of items with the color correct and with the color incorrect were also analyzed separately. The results were largely the same, with the exception that there was now substantial evidence for no main effect of age $\left(\mathrm{BF}_{10}=0.26\right)$ and no Age $\times$ Condition interaction $\left(\mathrm{BF}_{10}=\right.$ 0.20 ), rather than the inconclusive evidence found with $d^{\prime}$. The proportions of color correct items and $d^{\prime}$ for each group are presented in Table 2.

\section{Exploration of encoding time differences between groups}

In the present study, encoding time was not held constant between participants; instead, the encoding period lasted as long as it took the children to generate their stories. Exploratory analyses were conducted to determine whether encoding times differed between the different conditions and age groups. The mean encoding times across all stimuli for each age group and condition are summarized in Table 3 . The results were inconclusive regarding differences in encoding times between conditions $\left(\mathrm{BF}_{10}=0.97\right)$, between age groups $\left(\mathrm{BF}_{10}=0.45\right)$. However, there was support for no Condition $\times$ Age interaction $\left(\mathrm{BF}_{10}=0.18\right)$. Encoding time was not related to retrieval accuracy $\left(\mathrm{BF}_{10}=0.27\right)$, and when encoding time was added as a covariate in the main analyses, all results related to differences in retrieval accuracy remained the same.

Table 3 Encoding times for all groups in minutes, $M(S D)$

\begin{tabular}{ll}
\hline & Encoding Times \\
\hline 6-year-olds & \\
Unitized & $36.48(7.69)$ \\
Interactive & $33.00(11.82)$ \\
Non-unitization & $28.29(8.23)$ \\
8-year-olds & \\
Unitized & $37.22(10.28)$ \\
Interactive & $32.85(7.62)$ \\
Non-unitization & $34.98(9.43)$ \\
\hline
\end{tabular}

\section{Contributions of familiarity and recollection}

To determine whether the conditions differed in the contribution of familiarity, ROCs were constructed for individual participants and then averaged across conditions and age groups (see Fig. 1). For a review of ROC analyses, see Yonelinas and Parks (2007). ROCs compare the proportions of color correct to color incorrect responses at different levels of confidence. Participants' 3-point confidence judgments within each color were combined to create a 6-point scale ranging from Very confident yellow (1) to Very confident red (6). Analyses were run testing for differences in the proportions of confidence responses given at each level between age groups. No differences were found, all $\mathrm{BF}_{10} \mathrm{~S}<0.28$. The proportions of red and yellow responses were taken cumulatively for each confidence level, starting at 6 through confidence level 2 , for a total of 5 points. In this way, the cumulative proportion of yellow responses was plotted on the $x$-axis and the cumulative proportion of red responses was plotted on the $y$-axis, similar to the proportions of false alarms and hits plotted in traditional ROCs of recognition memory. Confidence levels of 1 are not plotted because they always result in a proportion of 1.0 for both cumulative red and yellow responses.

Linearity analysis Once individual plots were made, linearity analyses were performed. First, polynomial trend lines were fit to each individual's ROCs. The second-order coefficient values were recorded for each polynomial trend line $[M$ $(S D)$ : unitized $=-2.65(2.95)$, interactive $=-4.00(4.28)$, nonunitized $=-0.25(1.14)]$. According to the assumption that increasing the contribution of familiarity increases the curvature of the fit-line, the mean second-order coefficient served as an index of the level of familiarity contributing to retrieval. ${ }^{2}$ Model comparisons were run comparing a model with a main effect of condition to a null model to examine differences in the second-order polynomial term from the linearity analysis. The overall Bayesian ANOVA supported a difference between the conditions $\left(\mathrm{BF}_{10}=9,231 \cdot 22\right)$. Follow-up analysis showed decisive support for both the unitized and interactive conditions having greater curvature to their best-fit lines than did the non-unitization condition $\left(\mathrm{BF}_{10} \mathrm{~s}=1,749.15,11,578.57\right)$. However, differences between the unitized and interactive conditions were inconclusive.

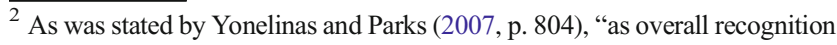
performance becomes very poor, the ROC approaches the chance diagonal, at which point it will necessarily have a slope of 1.0." Ratcliff, Sheu, and Gronlund (1992) caution against using linearity ROC analyses for $d^{\prime}$ less than 0.5 , which was clearly violated in our nonunitized condition. These findings beg the question of the legitimacy of the curvilinearity analysis for the nonunitized condition.
} 
Parameter estimates To complement the linearity analysis above, estimates of familiarity and recollection were derived by fitting a dual-process model of memory to the individual ROCs (Yonelinas, 1994, 1997). Under the dual-process model, the probability of getting a source correct response can be defined as the probability that an item is recollected as the target color plus the probability that it is not recollected as the target color, but is familiar enough to fall above the threshold level:

$P($ source correct $)=R_{t}+\left(1-R_{t}\right) \varphi\left(d^{\prime} / 2-c_{i}\right)$.

In the equation above, $R_{t}$ represents the recollection estimate of the target color (for the purposes of this study, red), $d^{\prime}$ represents the familiarity estimate, $c$ represents a specific criterion level (e.g., confidence level), and $\varphi$ is a function representing the cumulative proportion of responses exceeding a response criterion. The probability of a false alarm, however, is represented as the probability that an item is recollected as the lure color (for the purpose of this study, yellow), but is familiar enough to fall above the threshold level:

$P($ source incorrect $)=\left(1-R_{l}\right) \varphi\left(-d^{\prime} / 2 c_{i}\right)$.

These two equations can then be combined to give an overall representation of relational memory performance:

$P($ source correct $)-P($ source incorrect $)=R_{t}+\left(1-R_{t}\right) \varphi\left(d^{\prime} /\right.$ $\left.2 c_{i}\right)-\left(1-R_{l}\right) \varphi\left(-d^{\prime} / 2 c_{i}\right)$.

Using a sum-of-squares search algorithm, this model was fit to each individual's ROC points. The algorithm finds the best fit of the model by finding the parameters that result in the smallest sum-of-squares error, assuming variance in both hits and false alarms. Specifically, the algorithm finds the parameter estimates for the three free parameters $\left(R_{t}, R_{l}\right.$, and $\left.d^{\prime}\right)$ that minimize the distance between the observed known parameters (source correct rate, source incorrect rate, and criterion levels) and those predicted by the model. This results in probability recollection terms for each color that can vary from 0 to 1 , along with a $d^{\prime}$ familiarity term that typically varies from 0 to $4 .^{3}$ The recollection and familiarity terms were compared between age groups and conditions using model comparisons and Bayes factors (see Table 4).

For the estimates of familiarity, the effects of age and condition were compared against a null model, and their interaction was compared against a model with both main effects. We found decisive support for differences in the contribution of familiarity between conditions $\left(\mathrm{BF}_{10}=645,633,924\right)$, with support for differences between the two groups involving unitization and the non-unitization group $\left(\mathrm{BF}_{10} \mathrm{~S}=79,298,546\right.$ and $867,792,759$ ); however, the results were inconclusive regarding differences between unitized and interactive groups

\footnotetext{
$\overline{3}$ Although curvilinearity analysis may be untrustworthy for lowerperformance conditions, such concerns do not apply to the parameter estimation analyses. This type of method instead is known to be problematic when performance is very high, but we are unaware of similar cautions when performance is low (Yonelinas, 1999).
}

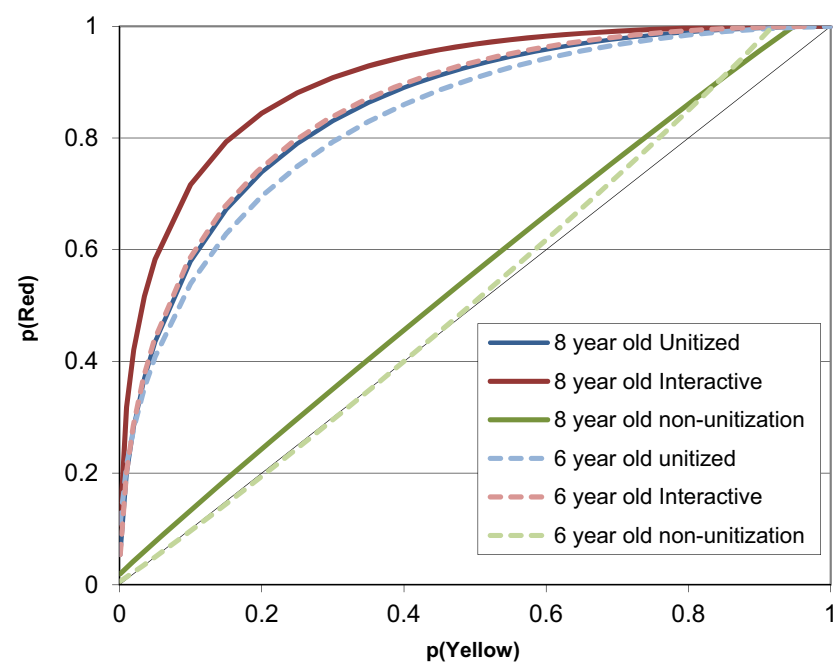

Fig. 1 ROC curves for each of the six conditions.

$\left(\mathrm{BF}_{10}=1.25\right)$. The results were also uninformative regarding the main effect of age $\left(\mathrm{BF}_{10}=0.52\right)$ and the Age $\times$ Condition interaction $\left(\mathrm{BF}_{10}=2.05\right)$.

For the estimates of recollection, the effects of age and condition and their interaction were compared against a model including only color and set as covariates. As with familiarity, we found support for a main effect of condition $\left(\mathrm{BF}_{10}=\right.$ 297,897,968). Follow-up analysis showed support for no differences between the unitized and interactive groups $\left(\mathrm{BF}_{10}=\right.$ 0.18); however, both of these groups showed a greater contribution of recollection than did the non-unitization group $\left(\mathrm{BF}_{10} \mathrm{~S}=595,235,715\right.$ and 297,897,968). There was substantial support for no difference between the age groups $\left(\mathrm{BF}_{10}=\right.$ $0.15)$ and no Age $\times$ Condition interaction $\left(\mathrm{BF}_{10}=0.20\right)$.

Because the contributions of both familiarity and recollection were found to be higher for both of the unitized groups (i.e., unitized and interactive) than for the non-unitization group, additional exploratory analysis was run to determine which memory component was responsible for the increase in memory performance.

For all three conditions, a dominance analysis was run to determine the contributions of familiarity, recollection for red items, and recollection for yellow items to memory performance $\left(d^{\prime}\right)$. Dominance analysis provides a qualitative assessment of the relative importances of all predictors in a multiple regression. The results provide a dominance score for each predictor, indicating its relative importance as compared to the other predictors. The sum of the dominance scores is equivalent to the $R^{2}$ of the model with all predictors included. One of the biggest strengths of this method is that it allows for comparison of the relative importances of multiple predictors in a regression framework (Azen \& Budescu, 2003), removing issues related to multicollinearity. Table 5 shows the overall $R^{2}$ for each regression, broken down into the portions that can be explained by the two predictors (recollection is 
Table 4 Estimates of familiarity and recollection, with recollection estimates collapsed across color, $M(S D)$

\begin{tabular}{lll}
\hline & Familiarity & Recollection \\
\hline 6-year-olds & & \\
Unitized & $0.97(0.74)$ & $0.21(0.20)$ \\
Interactive & $1.00(0.75)$ & $0.27(0.25)$ \\
Non-unitization & $0.07(0.15)$ & $0.03(0.05)$ \\
8-year-olds & & \\
Unitized & $0.98(0.66)$ & $0.26(0.24)$ \\
Interactive & $1.76(1.08)$ & $0.23(0.25)$ \\
Non-unitization & $0.12(0.18)$ & $0.05(0.10)$ \\
\hline
\end{tabular}

combined for red and yellow items). Larger values represent greater importance of that particular predictor in explaining memory performance. For all three conditions, familiarity was a more important predictor of memory performance than recollection for either color, and the importance of familiarity was greater for the two unitization conditions (unitized and interactive). This suggests that familiarity may play a larger role in memory performance in these conditions than recollection, and thus that the increase in memory performance with unitization can primarily be attributed to familiarity.

\section{Discussion}

The goal of the present study was to determine whether using visual unitization strategies would improve children's performance on a relational memory task through the increased contribution of familiarity. The results of the present study showed that, in fact, children who used visual unitization strategies (i.e., the unitized and interactive groups) performed better on the relation memory task and had higher contributions of familiarity than did the children who used the nonunitization visualization strategy. Additionally, along with greater contributions of familiarity, children who used unitization strategies also showed greater contributions of recollection at retrieval. However, a dominance analysis suggested that familiarity contributed to the increase in memory relatively more than did recollection.

Table 5 Results of the dominance analysis showing the relative importances of familiarity and recollection to memory performance, with recollection added across colors

\begin{tabular}{lll}
\hline & Familiarity & Recollection \\
\hline Unitized & .70 & .24 \\
Interactive & .77 & .13 \\
Non-unitization & .45 & .38 \\
\hline
\end{tabular}

Previous studies in adults have also shown significant differences in the contributions of familiarity for unitized and nonunitized conditions (Bastin et al., 2013; Diana et al., 2011; Diana et al., 2008; Rhodes \& Donaldson, 2008); however, the comparisons used in past adult work differed slightly from the comparisons used in the present study. For example, Rhodes and Donaldson (2008) used conditions similar to our interactive and non-unitization conditions, whereas Diana et al. (2011; Diana et al., 2008) and Bastin et al. (2013) compared the unitized condition used in the present study with a condition in which participants visualized a word and color being associated with each other. To our knowledge this is the first study to directly compare the unitized and interactive conditions in this manner, and although both were found to improve memory performance relative to a non-unitization condition, the results were inconclusive regarding whether one type of unitization is more effective than the other. Future research will be needed to determine differences in the levels of unitization between these and other conditions, to determine which manipulations lead to the greatest contribution of familiarity.

In contrast to most findings in adult studies, in addition to an increase in the contribution of familiarity with visual unitization, we also observed an increase in the contribution of recollection (cf. Parks \& Yonelinas, 2015). The proportional contribution of recollection, however, was much higher in the non-unitization condition, as would be expected. The three conditions were kept as similar a possible regarding the total time spent at encoding, length of a child's verbal response, and the number of items visualized; however, one primary difference between the two unitization conditions and the nonunitization condition was the generation of novel ideas versus the recitation of semantic knowledge. One potential concern regarding the present study, however, is the remarkably poor performance in the non-unitization condition. There are several potential explanations for this findings. First, the children in this study may not have had mature enough recollection processes to succeed at this task, since adults have been shown to perform above chance on similar conditions (i.e., Rhodes \& Donaldson, 2008). Second, generating stories for both of the unitization conditions was likely a more engaging and salient task, leading to more opportunities for deeper connections and relations to be formed, increasing the contribution of recollection. Even though performance in the non-unitization condition was low, this does not contradict the original hypothesis, and instead emphasizes how beneficial unitization strategies can be during early childhood. It should be noted, however, that due to the poor performance in this condition, the linearity analyses may not be reliable (Yonelinas \& Parks, 2007), and therefore future studies should aim to compare additional conditions under which the contribution of recollection would be more comparable to that in the unitization conditions. 
An additional surprising finding regarding recollection in the present study was the lack of a difference in the contribution of recollection between the two age groups. Previous studies have suggested that recollection abilities increase between 6 and 8 years of age (e.g., Ghetti \& Angelini, 2008; Sluzenski et al., 2006); however, no age-related differences were found in the present study. There are several potential explanations for why differences in recollection would not have been observed in the present study. One is that the age difference between the groups was not large enough. The present sample had an average age difference of slightly less than 2 years. Although the existing literature is consistent in the notion that familiarity develops before recollection, the precise age points at which the developmental trajectories deviate is unclear. A larger distance between the age groups might have revealed differences in recollection emerging; however, it would also have led to other methodological issues (e.g., greater differences in storytelling capabilities, confidence scale use, etc.). Another possible explanation for the lack of differences in recollection between the two groups is the task used in the present study. Many previous studies exploring the development of recollection have used easier tasks (e.g., having children remember more distinct pairings, such as animals on a unique background [Sluzenski et al., 2006] or toys and locations [Riggins, Rollins, \& Graham, 2013], rather than black-and-white pictures with arbitrary colored borders). Aside from being asked to visualize either the apple or the school bus, the children in the non-unitization condition did not have their attention drawn to the border, and therefore had little episodic context to recollect.

This study serves as an example of how empirical work in adults can be used to address applied questions within developmental research. We have taken current memory theory and methods commonly used in adults and used them to improve memory performance in children. There were, however, difficulties in applying these adult paradigms to a developmental sample. First, the task instructions had to be adjusted to match a 6-year-olds' vocabulary, potentially losing some of the nuanced differences between conditions when they are used with adults. For example, previous adult work compared our unitized condition to an associated condition (Diana, Yonelinas, \& Ranganath, 2007, 2008). We, however, were unable to come up with task instructions to explain the associated condition to children of this age, and therefore only the interactive condition was used. Additionally, because children are known to be overconfident in their memory abilities (Roebers, 2002), we had difficulty getting the younger children to use the entire confidence scale, making the ROCs and parameter estimates less reliable than they would be with adults.

Additionally, this was the first study to directly compare pure unitization and interactive imagery as visual unitization strategies. However, differences between these strategies were unclear, and therefore more research will be needed to determine what is necessary for unitization to occur and the benefits that can be observed. Potential methods for tapping into these subtle differences involve comparing conditions in adult populations that can understand minute differences in task instructions and using this comparison to explore neural substrates. Additionally, future studies should more closely examine specific details of the generated stories, in terms of distinctiveness, vividness, or meaningfulness, and how these factors relate to memory performance and to potential increases in familiarity.

Overall, the results of this study show that relational memory performance can be improved in early childhood through the use of visual unitization strategies, and that these strategies improve performance even more than separate-visualization strategies. Moreover, unitization increased the contribution of familiarity as well as recollection, and improvements in performance were predominantly attributable to increases in familiarity. This finding is particularly exciting in that it shows we can teach children strategies that make use of their earlier-developing familiarity processes (Billingsley et al., 2002; Brainerd et al., 2004; Ghetti \& Angelini, 2008). As we stated in the introduction, early childhood is a period of rapid development of relational memory abilities (Bemis \& Leichtman, 2013; Drummey \& Newcombe, 2002; Fandakova et al., 2013; Lloyd et al., 2009; Lorsbach \& Reimer, 2005; Riggins, 2014; Scarf et al., 2013; Yim et al., 2013); through the use of unitization strategies such as those in this study, we can help children succeed on these tasks while development is still occurring.

\section{References}

Azen, R., \& Budescu, D. V. (2003). The dominance analysis approach for comparing predictors in multiple regression. Psychological Methods, 8, 129-148. doi:10.1037/1082-989X.8.2.129

Bastin, C., Diana, R. A., Simon, J., Collette, F., Yonelinas, A. P., \& Salmon, E. (2013). Associative memory in aging: The effect of unitization on source memory. Psychology and Aging, 28, 275283. doi:10.1037/a0031566

Bemis, R. H., \& Leichtman, M. D. (2013). I remember when you taught me that! Preschool children's memories of realistic learning episodes. Infant and Child Development, 22, 603-621. doi:10.1002/ icd. 1807

Billingsley, R. L., Smith, M. L., \& McAndrews, M. P. (2002). Developmental patterns in priming and familiarity in explicit recollection. Journal of Experimental Child Psychology, 82, 251-277. doi:10.1016/S0022-0965(02)00007-3

Bower, G. H. (1970). Imagery as a relational organizer in associative learning. Journal of Verbal Learning and Verbal Behavior, 9, 529533. doi:10.1016/S0022-5371(70)80096-2

Brainerd, C. J., Holliday, R. E., \& Reyna, V. F. (2004). Behavioral measurement of remembering phenomenologies: So simple a child can do it. Child Development, 75, 505-522. doi:10.1111/j.1467-8624. 2004.00689.x

Craik, F. I. M. (2002). Levels of processing: Past, present and future? Memory, 10, 305-318. doi:10.1080/09658210244000135 
Cycowicz, Y. M., Friedman, D., \& Duff, M. (2003). Pictures and their colors: What do children remember? Journal of Cognitive Neuroscience, 15, 759-768. doi:10.1162/089892903322307465

Diana, R. A., Reder, L. M., Arndt, J., \& Park, H. (2006). Models of recognition: A review of arguments in favor of a dual-process account. Psychonomic Bulletin \& Review, 13, 1-21. doi:10.3758/ BF03193807

Diana, R. A., Van den Boom, W., Yonelinas, A. P., \& Ranganath, C. (2011). ERP correlates of source memory: Unitized source information increases familiarity-based retrieval. Brain Research, 1367, 278-286. doi:10.1016/j.brainres.2010.10.030

Diana, R. A., Yonelinas, A. P., \& Ranganath, C. (2007). Imaging recollection and familiarity in the medial temporal lobe: A threecomponent model. Trends in Cognitive Sciences, 11, 379-386. doi: 10.1016/j.tics.2008.03.001

Diana, R. A., Yonelinas, A. P., \& Ranganath, C. (2008). The effect of unitization on familiarity- based source memory: Testing a behavioral prediction derived from neuroimaging data. Journal of Experimental Psychology: Learning, Memory, and Cognition, 34, 730-740. doi:10.1037/0278-7393.34.4.730

Drummey, A. B., \& Newcombe, N. S. (2002). Developmental changes in source memory. Developmental Science, 5, 502-513. doi:10.1111/ 1467-7687.00243

Ecker, U. K. H., Maybery, M., \& Zimmer, H. D. (2013). Binding of intrinsic and extrinsic features in working memory. Journal of Experimental Psychology: General, 142, 218-234. doi:10.1037/ a0028732

Fandakova, Y., Shing, Y. L., \& Lindenberger, U. (2013). Differences in binding and monitoring mechanisms contribute to lifespan age differences in false memory. Developmental Psychology, 49, 18221832. doi: $10.1037 / \mathrm{a} 0031361$

Ghetti, S., \& Angelini, L. (2008). The development of recollection and familiarity in childhood and adolescence: evidence from the dualprocess signal detection model. Child Development, 79, 339-358. doi:10.1111/j.1467-8624.2007.01129.x

Ghetti, S., \& Bauer, P. J. (Eds.). (2012). Origins and development of recollection: Perspectives from psychology and neuroscience. New York: Oxford University Press.

Giovanello, K., Keane, M., \& Verfaellie, M. (2006). The contribution of familiarity to associative memory in amnesia. Neuropsychologia, 44, 1859-1865. doi:10.1016/j.neuropsychologia.2006.03.004

Hale, G. A., \& Piper, R. A. (1973). Developmental trends in children's incidental learning: Some critical stimulus differences. Developmental Psychology, 8, 327-335. doi:10.1037/h0034332

Jeffreys, H. (1961). The theory of probability (3rd ed.). Oxford: Oxford University Press, Clarendon Press.

Kan, I. P., Keane, M. M., Martin, E., Parks-Stamm, E. J., Lewis, L., \& Verfaellie, M. (2011). Implicit memory for novel associations between pictures: Effects of stimulus unitization and aging. Memory \& Cognition, 39, 778-790. doi:10.3758/s13421-011-0071-6

Kuo, T. Y., \& Van Petten, C. (2008). Perceptual difficulty in source memory encoding and retrieval: Prefrontal versus parietal electrical brain activity. Neuropsychologia, 46, 2243-2257. doi:10.1016/j. neuropsychologia.2008.02.018

Lloyd, M. E., Doydum, A. O., \& Newcombe, N. S. (2009). Memory binding in early childhood: Evidence for a retrieval deficit. Child Development, 80, 1321-1328. doi:10.1111/j.1467-8624.2009. 01353.x

Lorsbach, T. C., \& Reimer, J. F. (2005). Feature binding in children and young adults. Journal of Genetic Psychology, 166, 313-327. doi:10. 3200/GNTP.166.3.313-328

McGee, R. (1980). Imagery and recognition memory: The effects of relational organization. Memory \& Cognition, 8, 394-399. doi:10. 3758/BF03211135

Mitchell, K. J., Johnson, M. K., Raye, C. L., \& Green, E. J. (2004). Prefrontal cortex activity associated with source monitoring in working memory task. Journal of Cognitive Neuroscience, 16, 921-934. doi:10.1162/0898929041502724

Naveh-Benjamin, M. (2000). Adult-age differences in memory performance: Tests of an associative deficit hypothesis. Journal of Experimental Psychology: Learning, Memory, and Cognition, 26, 1170-1187. doi:10.1037/0278-7393.26.5.1170

Parks, C. M., \& Yonelinas, A. P. (2015). The importance of unitization for familiarity-based learning. Journal of Experimental Psychology: Learning, Memory, and Cognition, 41, 881-903. doi:10.1037/ $\mathrm{x} \operatorname{lm} 0000068$

Quamme, J. R., Yonelinas, A. P., \& Norman, K. A. (2007). Effect of unitization on associative recognition in amnesia. Hippocampus, 17, 192-200. doi:10.1002/hipo.20257

Ratcliff, R., Sheu, C., \& Gronlund, S. D. (1992). Testing global memory models using ROC curves. Psychological Review, 99, 518-535. doi: 10.1037/0033-295X.99.3.518

Rhodes, S. M., \& Donaldson, D. I. (2007). Electrophysiological evidence for the influence on the unitization processes engaged during episodic retrieval: Enhancing familiarity based remembering. Neuropsychologia, 45, 412-424. doi:10.1016/j.neuropsychologia. 2006.06.022

Rhodes, S. M., \& Donaldson, D. I. (2008). Electrophysiological evidence for the effect of interactive imagery on episodic memory: Encouraging familiarity for non-unitized stimuli during associative recognition. NeuroImage, 39, 873-884. doi:10.1016/j.neuroimage. 2007.08.041

Riggins, T. (2014). Longitudinal investigation of source memory reveals different trajectories for item memory and binding. Developmental Psychology, 50, 449-459. doi:10.1037/a0033622

Riggins, T., Rollins, L., \& Graham, M. (2013). Electrophysiological investigation of source memory in early childhood. Developmental Neuropsychology, 38, 180-196. doi:10.1080/87565641.2012. 762001

Roebers, C. M. (2002). Confidence judgments in children's and adult's event recall and suggestibility. Developmental Psychology, 38, 1052-1067.

Roebers, C. M., Gelhaar, T., \& Schneider, W. (2004). "It's magic!” The effects of presentation modality on children's event memory, suggestibility, and confidence judgments. Journal of Experimental Child Psychology, 87, 320-335. doi:10.1016/j.jecp.2004.01.004

Rouder, J. N., Morey, R. D., Speckman, P. L., \& Province, J. M. (2012). Default Bayes factors in ANOVA designs. Journal of Mathematical Psychology, 56, 356-374. doi:10.1016/j.jmp.2012.08.001

Ryan, E. B., Ledger, G. W., \& Weed, K. A. (1987). Acquisition and transfer of an integrative imagery strategy by young children. Child Development, 58, 443-452. doi:10.1111/j.1467-8624.1987. tb01392.x

Ryan, J. D., Moses, S. N., Barense, M., \& Rosenbaum, R. S. (2013). Intact learning of new relations in amnesia as achieved through unitization. Journal of Neuroscience, 33, 9601-9613. doi:10.1523/ JNEUROSCI.0169-13.2013

Scarf, D., Gross, J., Colombo, M., \& Hayne, H. (2013). To have and to hold: Episodic memory 3- and 4-year-old children. Developmental Psychobiology, 55, 125-132. doi:10.1002/dev.21004

Schneider, W., \& Sodian, B. (1997). Memory strategy development: Lessons from longitudinal research. Developmental Review, 17, 442-461. doi:10.1006/drev.1997.0441

Sluzenski, J., Newcombe, N. S., \& Kovacs, S. L. (2006). Binding, relational memory, and recall of naturalistic events: A developmental perspective. Journal of Experimental Psychology: Learning, Memory, and Cognition, 32, 89-100. doi:10.1037/0278-7393.32.1. 89

Snodgrass, J. G., \& Vanderwart, M. (1980). A standardized set of 260 pictures: Norms for name agreement, image agreement, familiarity, and visual complexity. Journal of Experimental Psychology: 
Human Learning and Memory, 6, 174-215. doi:10.1037/20787393.6.2.174

Spiker, C. C., \& Cantor J. H. (1980). The effects of stimulus type, training, and chronological age on children's identification and recording of multidimensional stimuli. Journal of Experimental Child Psychology, 30, 144-158. doi:10.1016/0022-0965(80)90081-8

Tibon, R., Gronau, N., Scheuplein, A. L., Mecklinger, A., \& Levy, D. A. (2014). Associative recognition processes are modulated by the semantic unitizability of memoranda. Brain and Cognition, 92C, 19 31. doi:10.1016/j.bandc.2014.09.009

Wilton, R. N. (1989). The structure of memory: Evidence concerning the recall of surface and background colour of shapes. Quarterly Journal of Experimental Psychology, 41A, 579-598. doi:10.1080/ 14640748908402383

Yim, H., Dennis, S. J., \& Sloutsky, V. M. (2013). The development of episodic memory: Items, contexts, and relations. Psychological Science, 24, 2163-2172. doi:10.1177/0956797613487385

Yonelinas, A. P. (1994). Receiver operating characteristics in recognition memory: Evidence for a dual-process model. Journal of Experimental Psychology: Learning, Memory, and Cognition, 20, 1341-1354. doi:10.1037/0278-7393.20.6.1341

Yonelinas, A. P. (1997). Recognition memory ROCs for item and associative information: The contribution of recollection and familiarity. Memory \& Cognition, 25, 747-763. doi:10.3758/BF03211318
Yonelinas, A. P. (1999). The contribution of recollection and familiarity to recognition and source-memory judgments: A formal dual-process model and an analysis of receiver operating characteristics. Journal of Experimental Psychology: Learning, Memory, and Cognition, 25, 1415-1434. doi:10.1037/0278-7393.25.6.1415

Yonelinas, A. P. (2002). The nature of recollection and familiarity: A review of 30 years of research. Journal of Memory and Language, 46, 441-517. doi:10.1006/jmla.2002.2864

Yonelinas, A. P., Kroll, N. E., Dobbins, I. G., \& Soltani, M. (1999). Recognition memory for faces: When familiarity supports associative recognition judgments. Psychonomic Bulletin \& Review, 6, 654-661. doi:10.3758/BF03212975

Yonelinas, A. P., \& Parks, C. M. (2007). Receiver operating characteristics (ROCs) in recognition memory: A review. Psychological Bulletin, 133, 800-832. doi:10.1037/0033-2909.133.5.800

Zheng, Z., Li, J., Xiao, F., Broster, L. S., \& Jiang, Y. (2015). Electrophysiological evidence for the effects of unitization on associative recognition memory in older adults. Neurobiology of Learning and Memory, 121, 59-71.

Zheng, Z., Li, J., Xiao, F., Ren, W., \& He, R. (2016). Unitization improved source memory in older adults: An event-related potential study. Neuropsychologia, 89, 232-244. doi:10.1016/j. neuropsychologia.2016.06.025 